\title{
Acasalamento entre Ovelhas Deslanadas e Reprodutores Especializados para Corte: Desempenho Produtivo até a Desmama
}

\author{
Rui Machado', Aurino Alves Simplício², Maria Eliza Barbieri²
}

\begin{abstract}
RESUMO - O presente experimento foi realizado para avaliar o desempenho produtivo de ovelhas deslanadas do Nordeste do Brasil acasaladas com machos de raças especializadas para corte. Dados de 305 parições oriundas de 134 matrizes dentro de três períodos de monta foram usados para determinar as taxas de desmame e sobrevivência das crias, o peso vivo ao desmame e o peso vivo total desmamado por ovelha acasalada. As fêmeas foram acasaladas, em sistema de rodízio de uso dos machos das raças Santa Inês, Hampshire-Down, Ile-de-France, Suffolk e Texel. O peso das crias ao desmame não diferiu entre genótipos. A taxa de desmame foi mais baixa para crias de Suffolk e de Texel, ao passo que crias de Hampshire-Down e Ile-de-France tiveram as taxas de sobrevivência mais baixas. Houve efeito compensatório entre os pesos ao nascer e ao desmame e as taxas de sobrevivência e desmame, de modo que o peso total de cordeiro desmamado não diferiu entre genótipos, à exceção para o grupo $\mathrm{F}_{1}$ de Hampshire-Down, devido à baixa taxa de sobrevivência dos cordeiros (71,4\%), se comparado com as crias de Ile-de-France. Os acasalamentos ocorridos durante a estação chuvosa foram responsáveis pela menor produtividade das ovelhas. O desempenho das crias Santa Inês comparou-se ao das raças especializadas para o corte, podendo ser indicada como raça parental em programas de cruzamento com ovelhas deslanadas do Nordeste do Brasil.
\end{abstract}

Palavras-chave: ovino, cruzamento, desmama

\section{Hairsheep Females Mated to Specialized Meat-Type Rams: Productive Performance up to Weaning}

\begin{abstract}
This trial was conducted to evaluate production levels of native breed ewes of Northeast Brazil mated to rams of specialized meat-type breeds. A total of 305 lambings from 134 ewes over three periods of mating were used to determine weaning rate, survival rate and live weight at weaning and total weight of weaned lamb. The hairsheep ewes were mated in a rotational scheme of the use of rams from Santa Inês, Hampshire-Down, Ile-de-France, Suffolk and Texel breeds. Live weight at weaning did not differ among lamb genotypes. Weaning rate was lower for Suffolk and Texel crossbred lambs. Whereas, the lowest survival rates were observed for Hampshire-Down and Ile-de-France crossbred lambs. There was a compensatory effect among the weight at birth, weaning weight; weaning and survival rate, so that the total weight of weaned lamb did not differ among lamb genotypes except for Hampshire Down crossbred, due to its lower survival rate (71.4\%) as compared to Ile-de-France crossbred lambs. The mating throughout the rainy season accounted for the decreased levels of productivity of the ewes. The Santa Inês hairsheep lamb performance compared to the other specialized meat-type sheep breeds and could be indicated as ram breed in crossbreeding programs for hairsheep in Northeast Brazil.
\end{abstract}

Key Words: sheep, crossbreeding, weaning

\section{Introdução}

Visto que a capacidade de produção dos ovinos está condicionada não somente ao meio, mas também a fatores genéticos, o melhoramento ambiental deve ser acompanhado pelo genético, uma vez que, mesmo sob condições ótimas, animais de genótipo inferior terão baixa produção (GUERREIRO, 1989). O cruzamento de ovelhas nativas do Nordeste do Brasil (SRD) com carneiros especializados para corte é alternativa para aumentar a produção de carne, pois faz uso de animais "híbridos", propiciando o nascimento de cordeiros mais resistentes e com maior velocidade de crescimento. Além disso, os animais tipo "cruza" têm maior capacidade para atender as demandas de mercado por carcaças maiores, mais pesadas, musculosas, com pouca gordura e carne macia, provenientes de animais mais jovens ao abate (CARDELLINO, 1989). Assim, é mister conhecer o desempenho produtivo de fêmeas nativas acasaladas com machos de raças especializadas, mantidos sob condições climáticas adversas, motivo pelo qual se desenvolveu este trabalho. 


\section{Material e Métodos}

O trabalho foi conduzido na fazenda experimental da EMBRAPA-CNPC, localizada no município de Sobral, Estado do Ceará. Esta região semi-árida encontra-se a $3^{\circ} 42^{\prime}$ de latitude Sul e $40^{\circ} 21^{\prime}$ de longitude Oeste, com altitude de 83 metros e clima do tipo Aw de Savana pela classificação de Köppen (MACHADO e SIMPLÍCIO, 1998), tendo uma estação seca, geralmente de julho a dezembro, e outra chuvosa, de janeiro a junho. A temperatura média anual é de $28^{\circ} \mathrm{C}(22 \mathrm{e}$ $35^{\circ} \mathrm{C}$, como médias mínima e máxima, respectivamente) e umidade relativa do ar de $69 \%$. Os valores da precipitação pluvial ( $\mathrm{mm}$ ) observados nesta localidade durante o período experimental foram descritos por MACHADO e SIMPLÍCIO (1998).

Foram utilizados os dados de 305 parições, provenientes de 134 ovelhas deslanadas, pluríparas, do tipo sem raça definida (SRD). Foram estabelecidas três estações de monta e, por conseguinte, três de nascimento e três de desmama, conforme a Tabela 1. A duração do período de monta foi pré-estabelecida em 85 dias ou até 17 dias depois que todas as ovelhas expostas haviam sido acasaladas por, pelo menos, uma oportunidade (Tabela 1). Assim, a duração efetiva dos períodos de monta foi de 79,56 e 39 dias, respectivamente. Cinco rufiões auxiliaram na detecção do estro e as ovelhas foram acasaladas em sistema de rodízio entre cinco carneiros adultos, sendo um Hampshire Down, um Ile-de-France, um Suffolk, um Texel e um Santa Inês, este último usado como controle. Este sistema de acasalamento permitiu que a distribuição de fêmeas entre os reprodutores fosse, aproximadamente, igual.

As matrizes foram manejadas semi-intensivamente, recebendo suplementação alimentar, volumosa e concentrada, no período seco do ano, no terço final da prenhez e durante a lactação.

O desmame dos cordeiros ocorreu aos 84 dias de idade. Findo o desmame correspondente a cada estação de nascimento, foram computados: 1) número de ovelhas acasaladas; 2) número de ovelhas paridas; 3 ) número de cordeiros nascidos; e 4) número de cordeiros desmamados. Foram mensurados peso vivo dos cordeiros ao nascer (kg) e peso vivo dos cordeiros aos $28,56,84$ e 112 dias $(\mathrm{kg})$. As seguintes taxas foram determinadas (FERNANDES et al., 1986):

$$
\begin{aligned}
& \text { Desmama }=\frac{\mathrm{N}^{\mathrm{o}} \text { cordeiros desmamados } \times 100}{\mathrm{~N}^{\mathrm{O}} \text { ovelhas acasaladas }} \\
& \text { Sobrevivência }=\frac{\mathrm{N}^{\mathrm{o}} \text { cordeiros desmamados } \mathrm{x} 100}{\mathrm{~N}^{\mathrm{O}} \text { cordeiros nascidos }}
\end{aligned}
$$

e calculado:

Peso desmamado $=$ Total $\mathrm{kg}$ de cordeiro desmamado

$$
\mathrm{N}^{0} \text { de ovelhas paridas }
$$

As taxas obtidas foram tratadas como proporções e, em seguida, analisadas usando o modelo para dados categóricos - CATMOD do SAS (1990). Este modelo analisa dados que podem ser expressos em tabelas de contingência bi-dimensionais. Assim, os efeitos das cinco raças de padreadores foram comparados dois-a-dois. Similarmente, contrastes binários também foram usados para comparação entre os efeitos das três épocas de nascimento. Os resultados relativos a pesos vivos foram submetidos à análise de variância e, quando necessário, submetidos ao teste LSD (STEEL e TORRIE, 1980). Estas análises basearam-se no método dos quadrados mínimos, utilizando-se o procedimento GLM (SAS, 1990). O modelo matemático empregado para peso vivo dos cordeiros ao nascer teve a inclusão da covariável peso da matriz ao parto, sendo representado por:

$$
\begin{aligned}
& \mathrm{Y}_{\mathrm{ijklmn}}=\mu+\mathrm{R}_{\mathrm{i}}+\mathrm{S}_{\mathrm{j}}+\mathrm{T}_{\mathrm{k}}+\mathrm{X}_{1}+\mathrm{RX}_{\mathrm{il}}+\mathrm{b}\left(\mathrm{P}_{\mathrm{ijklm}}-\overline{\mathrm{P}}\right)+\mathrm{E}_{\mathrm{ijklmn}} \\
& \text { em que } \\
& \mathrm{Y}_{\mathrm{ijklmn}}=\text { peso ao nascer; } \\
& \mu \quad=\text { média geral; } \\
& \mathrm{R}_{\mathrm{i}}=\text { efeito da } \mathrm{i}_{\text {ésima }} \text { raça de reprodutor, }
\end{aligned}
$$
$\mathrm{i}=1,2,3,4,5$;

$\mathrm{S}_{\mathrm{j}}=$ efeito da $\mathrm{j}_{\text {ésima }}$ época de nascimento, $\mathrm{j}=1,2,3$;

$\mathrm{T}_{\mathrm{k}} \quad=$ efeito do $\mathrm{k}_{\text {ésimo }}$ tipo de parto, $\mathrm{k}=1,2 ;$

$\mathrm{X}_{1} \quad=$ efeito da $1_{\text {ésimo }}$ sexo da cria, $1=1,2$;

$\mathrm{RX}_{\mathrm{il}}=$ efeito da interação raça do reprodutor vs. Sexo da cria, il = 1,2,3,4,5,6,7,8,9,10;

$\mathrm{b}=$ coeficiente de regressão linear do peso vivo ao nascer $\left(\mathrm{Y}_{\mathrm{ijklmn}}\right)$ em função do peso vivo da matriz ao parto;

$\mathrm{P}_{\mathrm{ijklm}}=$ peso vivo da matriz ao parto;

$\overline{\mathrm{P}}=$ média do peso vivo das matrizes ao parto; e

$E_{\mathrm{ijklmn}}=$ erro aleatório associado a cada observação suposto normal e independentemente distribuído com média zero e variância $\sigma^{2}$.

Os pesos vivos das crias aos 28, 56, 84 e 112 dias foram analisados segundo o exemplo de modelo descrito abaixo.

$$
\begin{aligned}
& \mathrm{Y}_{\mathrm{ijklmn}}=\mu+\mathrm{R}_{\mathrm{i}}+\mathrm{S}_{\mathrm{j}}+\mathrm{T}_{\mathrm{k}}+\mathrm{X}_{1}+\mathrm{RX}_{\mathrm{il}}+\mathrm{b}\left(\mathrm{P}_{\mathrm{ijklm}}-\overline{\mathrm{P}}\right)+\mathrm{E}_{\mathrm{ijklmn}} \\
& \text { em que } \\
& \mathrm{Y}_{\mathrm{ijklmn}}=\text { peso aos } 28 \text { dias; } \\
& \mu \quad \text { média geral; } \\
& \mathrm{R}_{\mathrm{i}}=\text { efeito da } \mathrm{i}_{\text {ésima }} \text { raça de reprodutor, } \\
& \mathrm{i}=1,2,3,4,5 ;
\end{aligned}
$$


708 Rev. bras. zootec.

$\mathrm{S}_{\mathrm{j}} \quad=$ efeito daj $_{\text {ésima }}$ época denascimento, $\mathrm{j}=1,2,3$;

$\mathrm{T}_{\mathrm{k}} \quad=$ efeito do $\mathrm{k}_{\text {ésimo }}$ tipo de parto, $\mathrm{k}=1,2 ;$

$\mathrm{X}_{1} \quad=$ efeito da $1_{\text {ésimo }}$ sexo da cria, $1=1,2$;

$\mathrm{RX}_{\mathrm{il}}=$ efeito da interação raça do reprodutor vs. Sexo da cria, il = 1,2,3,4,5,6,7,8,9,10;

b = coeficiente de regressão linear do peso vivo aos 28 dias ( $\mathrm{Y}_{\mathrm{ijklmn}}$ ) em função do peso vivo da matriz ao parto;

$\mathrm{P}_{\mathrm{ijklm}}=$ peso vivo da matriz ao parto;

$\overline{\mathrm{P}}=$ média do peso vivo das matrizes ao parto; e

$\mathrm{E}_{\mathrm{ijklmn}}=$ erro aleatório associado a cada observação, suposto normal e independentemente distribuído com média zero e variância $\sigma^{2}$.

Para peso total de cordeiro desmamado, empregou-se o modelo:

$\mathrm{Y}_{\mathrm{ijklm}}=\mu+\mathrm{R}_{\mathrm{i}}+\mathrm{S}_{\mathrm{j}}+\mathrm{T}_{\mathrm{k}}+\mathrm{b}\left(\mathrm{P}_{\mathrm{ijkl}}-\overline{\mathrm{P}}\right)+\mathrm{E}_{\mathrm{ijklm}}$ em que

$\mathrm{Y}_{\mathrm{ijklm}}=$ peso total de cordeiro desmamado;

$\mu \quad=$ média geral;

$\mathrm{R}_{\mathrm{i}}=$ efeito da $\mathrm{i}_{\text {ésima }}$ raça de reprodutor, $\mathrm{i}=1,2,3,4,5$;

$\mathrm{S}_{\mathrm{j}}=$ efeito da $\mathrm{j}_{\text {ésima }}$ época de nascimento, $\mathrm{j}=1,2,3$;

$\mathrm{T}_{\mathrm{k}} \quad=$ efeito do $\mathrm{k}_{\text {ésimo }}$ tipo de parto, $\mathrm{k}=1,2 ;$

$\mathrm{b}=$ coeficiente de regressão linear do peso total desmamado $\left(\mathrm{Y}_{\mathrm{ijklmn}}\right)$ em função do peso vivo da matriz ao parto;

$\mathrm{P}_{\mathrm{ijkl}}$ = peso vivo da matriz ao parto;

$\overline{\mathrm{P}}=$ média do peso vivo das matrizes ao parto; e

$\mathrm{E}_{\mathrm{ijklm}}=$ erro aleatório associado a cada observação, suposto normal e independentemente distribuído com média zero e variância $\sigma^{2}$.

\section{Resultados e Discussão}

Pela análise da Tabela 2, pode-se inferir que os efeitos da raça do padreador sobre a eficiência produtiva das ovelhas foram significativos $(\mathrm{P}<0,05)$ para as taxas de desmame e sobrevivência das crias, bem como para o peso ao nascer dos cordeiros. As diferenças raciais observadas no peso ao nascer concordam com os achados de CASOLI et al. (1984), McEWAN e HANRAHAN (1986), FALAGAN e GARCIA DE SILES (1986), CONSTANTINOU e MAVROGENIS (1987) e FERNANDES e DESHMUKH (1988). Em adição, SILVA (1990) relata que o padreador é fonte de variação significativa do peso vivo ao nascer das crias.

O peso vivo ao desmame não variou significativamente em função da raça parental, concordando com os achados de BUIRAS et al. (1986) para cordeiros cruza, e invalidando, dentro das condições semiáridas do Ceará, a recomendação taxativa feita por GUNEY (1990), que apregoava o uso exclusivo de Ile-de-France como raça parental em sistemas de cruzamento industrial. Os cordeiros $\mathrm{F}_{1}$ de raças

Tabela 1- Época de monta, nascimento e desmama Table 1 - Season of mating, lambing and weaning

\begin{tabular}{|c|c|c|}
\hline \multicolumn{3}{|c|}{ Estação } \\
\hline & Season & \\
\hline Monta & Parição & Desmama \\
\hline Mating & Lambing & Weaning \\
\hline$\overline{\mathrm{abr} / 88-\mathrm{jul} / 88}$ & set - nov $/ 88$ & $\operatorname{dez} / 88-\mathrm{fev} / 89$ \\
\hline $\mathrm{dez} / 88-\mathrm{fev} / 89$ & mai-jul/89 & ago/89 - out/89 \\
\hline out $/ 89-\operatorname{dez} / 89$ & $\operatorname{mar}-\mathrm{abr} / 90$ & jun/90 - jul/90 \\
\hline
\end{tabular}

Tabela 2 - Efeito da raça do reprodutor sobre a eficiência produtiva de ovelhas deslanadas do tipo sem raça definida Table 2- Effect of breed of the sire on the productive performance of native (SRD) hairsheep ewes

\begin{tabular}{|c|c|c|c|c|c|c|c|c|}
\hline \multirow[t]{2}{*}{$\begin{array}{l}\text { Raça } \\
\text { Breed }\end{array}$} & \multicolumn{2}{|c|}{$\begin{array}{c}\text { Taxa }(\%) \\
\text { Rate }\end{array}$} & \multirow[t]{2}{*}{$\mathrm{N}$} & \multicolumn{2}{|c|}{$\begin{array}{l}\text { Peso das crias } \\
\text { Lamb weight }\end{array}$} & \multirow{2}{*}{$\begin{array}{c}\text { Ganho de peso } \\
\text { no aleitamento }(\mathrm{g} / \mathrm{d}) \\
\text { Daily gain } \\
\text { at suckling }\end{array}$} & \multicolumn{2}{|c|}{$\begin{array}{c}\text { Peso total } \\
\text { de cordeiro }(\mathrm{kg})\end{array}$} \\
\hline & $\begin{array}{c}\text { Desmama } \\
\text { Weaning }\end{array}$ & $\begin{array}{c}\text { Sobrevivência } \\
\text { Survival }\end{array}$ & & $\begin{array}{l}\text { Ao nascer } \\
\text { At birth }\end{array}$ & $\begin{array}{l}\text { Ao desmame } \\
\text { At weaning }\end{array}$ & & $\begin{array}{c}\text { Nascido } \\
\text { Born }\end{array}$ & $\begin{array}{c}\text { Desmamado } \\
\text { Weaned }\end{array}$ \\
\hline$\overline{\text { Santa Inês }}$ & $88,7^{\mathrm{b}}$ & $89,0^{\mathrm{a}}$ & 81 & $3,22^{c}$ & 11,4 & 97,9 & 5,7 & $12,5^{\mathrm{ab}}$ \\
\hline Hamp. Down & $96,0^{\mathrm{a}}$ & $71,4^{b}$ & 84 & $3,73^{b}$ & 12,5 & 105,5 & 5,8 & $11,2^{b}$ \\
\hline Ile-de-France & $86,6^{\mathrm{ab}}$ & $80,7^{\mathrm{ab}}$ & 89 & $3,79^{\mathrm{ab}}$ & 13,1 & 112,4 & 5,8 & $14,4^{\mathrm{a}}$ \\
\hline Suffolk & $74,3^{\mathrm{c}}$ & $84,8^{\mathrm{a}}$ & 66 & $3,95^{\mathrm{a}}$ & 12,7 & 105,4 & 5,8 & $13,6^{\mathrm{ab}}$ \\
\hline Texel & $70,4^{\mathrm{c}}$ & $90,2^{\mathrm{a}}$ & 41 & $3,82^{\mathrm{ab}}$ & 14,1 & 124,0 & 5,8 & $13,9^{\mathrm{ab}}$ \\
\hline
\end{tabular}

Médias, na coluna, seguidas de letras diferentes são diferentes $(P<0,05)$ pelo teste LSD.

Means, within a column, followed by different letters are different $(P<.05)$ by LSD test. 
exóticas tiveram ganhos de peso superiores a $100 \mathrm{~g}$ diários e atingiram pesos ao desmame maiores, embora não estatisticamente superiores aos obtidos pelas crias de Santa Inês. Em estudos prévios, os efeitos de genótipo foram significativos, quando foram comparados entre si os tipos raciais: DorsetDown, Ile-de-France, South-Down e Suffolk cruzados com Apenino (CASOLI et al., 1984); Texel e Suffolk em estado de pureza (McEWAN e HANRAHAN, 1986); Mutton Merino, Charmoise, Ile-de-France e Texel cruzados com Aragon (FALAGAN e GARCIA DE SILES, 1986); e cruzas de Suffolk e HampshireDown (ABOUL-NAGA e ABOUL-ELA, 1987). Assim, no período de aleitamento, não se expressaram as diferenças genotípicas esperadas, pois a curva de crescimento do cordeiro na fase de aleitamento acompanha a curva de lactação da matriz (FIGUEIRÓ, 1989), que está mais intimamente ligada à oferta nutricional durante a lactação que ao genótipo da cria.

A Tabela 2 permite inferir certa compensação entre as taxas de desmame e as de sobrevivência de crias, dentro da raça parental. Em conseqüência, o peso total de cordeiro desmamado não diferiu estatisticamente entre as raças, exceto para a $\mathrm{F}_{1}$ Hampshire Down, que, devido à baixa taxa de sobrevivência de crias, teve menor peso total desmamado, quando comparada com as crias de Ile-de-France. Por outro lado, o peso total de cordeiros desmamados meio- sangue Texel e Suffolk foi equivalente ao peso dos animais cruza das demais raças. Este resultado pode ser atribuído ao elevado peso ao desmame destas crias. As menores taxas de desmame observadas para Suffolk e Texel, como reflexo de menor número de ovelhas paridas, foram compensadas por taxas elevadas de sobrevivência de crias. Assim, o peso total desmamado foi equivalente ao de outros grupos raciais com melhor desempenho em desmama (Hampshire-Down, Santa Inês e Ile-de-France) ou em sobrevivência (Santa Inês).

A Tabela 3 apresenta o desenvolvimento ponderal dos cordeiros lactentes e a sua viabilidade. Os pesos tomados ao nascer ou ao desmame e a mortalidade de cordeiros neste intervalo são características influenciadas pelo genótipo (CASOLI et al., 1984; McEWAN e HANRAHAN, 1986; FALAGAN e GARCIA DE SILES, 1986; CONSTANTINOU e MAVROGENIS, 1987; e FERNANDES e DESHMUKH, 1988).

Aparentemente, a elevada mortalidade de crias influenciou negativamente o peso total de cordeiros desmamados meio-sangue Hampshire-Down (Tabela 2).

CONSTANTINOU e MAVROGENIS (1987) verificaram que as crias tipo cruza apresentaram maior taxa de sobrevivência, sendo que seus pesos ao nascer e ao desmame tiveram heterose de 5,7 e 9,8\%, respectivamente. O crescimento e a mortalidade das crias foram significativamente influenciados pela raça

Tabela 3 - Peso vivo médio $(\mathrm{kg})$ e taxa de mortalidade de cordeiros $F_{1}$ de acordo com a raça parental e o sexo ${ }^{1}$

Table 3 - Live weight $(\mathrm{kg})$ and mortality of $F_{1}$ crossbred lambs according to breed of sire and sex ${ }^{1}$

\begin{tabular}{|c|c|c|c|c|c|c|}
\hline \multirow{3}{*}{$\begin{array}{l}\text { Raça } \\
\text { Breed }\end{array}$} & \multicolumn{5}{|c|}{ Peso vivo (kg) } & \multirow{3}{*}{$\begin{array}{c}\text { Mortalidade (\%) } \\
\text { Mortality }\end{array}$} \\
\hline & & \multicolumn{3}{|c|}{ Live weight } & \multirow[b]{2}{*}{$\begin{array}{c}112 \text { dias } \\
\text { days }\end{array}$} & \\
\hline & $\begin{array}{r}\text { Nascer } \\
\text { Birth } \\
\end{array}$ & $\begin{array}{c}28 \text { dias } \\
\text { days }\end{array}$ & $\begin{array}{c}56 \text { dias } \\
\text { days }\end{array}$ & $\begin{array}{c}84 \text { dias } \\
\text { days }\end{array}$ & & \\
\hline \multicolumn{7}{|l|}{ Macho } \\
\hline \multicolumn{7}{|l|}{ Ram lamb } \\
\hline Santa Inês & 3,38 & 7,32 & 9,63 & 12,68 & 16,27 & 17,02 \\
\hline Hampshire-Down & n 3,79 & 7,11 & 9,34 & 11,60 & 14,91 & 26,67 \\
\hline Ile-de-France & 3,72 & 7,75 & 10,11 & 12,65 & 15,78 & 26,32 \\
\hline Suffolk & 4,10 & 8,28 & 10,89 & 12,77 & 17,22 & 21,21 \\
\hline Texel & 3,97 & 9,18 & 11,53 & 16,22 & 20,23 & 9,52 \\
\hline \multicolumn{7}{|l|}{ Fêmea } \\
\hline \multicolumn{7}{|l|}{ Ewe lamb } \\
\hline Santa Inês & 3,02 & 8,01 & 8,40 & 9,98 & 13,11 & 2,86 \\
\hline Hampshire Down & 3,70 & 6,61 & 9,58 & 13,07 & 18,62 & 29,63 \\
\hline Ile-de-France & 3,85 & 7,26 & 10,37 & 13,40 & 17,27 & 14,00 \\
\hline Suffolk & 3,81 & 6,99 & 10,16 & 12,63 & 17,47 & 9,09 \\
\hline Texel & 3,67 & 6,71 & 9,77 & 11,91 & 18,41 & 10,00 \\
\hline $\mathrm{CV}(\%)$ & 17,19 & 61,64 & 21,20 & 18,91 & 16,37 & 23,68 \\
\hline
\end{tabular}

${ }_{1}^{1}$ Procedimentos para comparação múltipla entre os valores não foram executados. 
parental exótica em um experimento conduzido no Egito por ABOUL-NAGA e ABOUL-ELA (1987). As cruzas de Suffolk foram superiores às crias de Hampshire-Down.

A Tabela 4 mostra os efeitos da época de acasalamento sobre alguns parâmetros produtivos. Cumpre lembrar que MACHADO e SIMPLÍCIO (1998) haviam verificado que a pluviosidade durante a execução do trabalho teve comportamento distinto "entre anos" e "entre épocas", refletindo, possivelmente, na disponibilidade de forragens e, conseqüentemente, na oferta alimentar ao rebanho, influenciando os pesos das matrizes à cobrição e ao parto, bem como a produção leiteira das mesmas. Entretanto, nenhum dos anos em que o trabalho se desenvolveu pode ser considerado atípico.

As maiores taxas de desmame e sobrevivência de crias foram observadas quando os partos ocorreram no período de março a abril de 1990, podendo ser explicadas pela curva da precipitação pluvial daquele ano (MACHADO e SIMPLÍCIO, 1998). Naquela ocasião, o pico de chuvas antecedeu o pico dos nascimentos e a distribuição das precipitações em 1990 foi, razoavelmente, homogênea durante a estação chuvosa, com pequenos gradientes entre meses. Esta situação favoreceu a obtenção de cordeiros mais pesados que em outras épocas, por explorar ao máximo a típica velocidade de crescimento (ganho de peso diário) deste estádio da vida do ruminante (FIGUEIRÓ, 1989).

Em concordância com os achados de SEEGERS e DENIS (1982), as altas temperaturas ambientais durante o terço final da prenhez (setembro a novembro/88) produziram cordeiros com menores pesos ao nascer. Possivelmente, houve redução do consumo de matéria seca pelas matrizes durante as épocas mais quentes (THERIEZ et al., 1987). Entretanto, a magnitude da ação negativa do calor não foi suficiente para interferir na manutenção da prenhez
(ENCARNAÇÃO, 1986), sendo verificadas elevadas taxas de desmame, mesmo para cordeiros nascidos na época mais quente do ano. FIGUEIRÓ (1989) já havia afirmado que as exigências nutricionais de uma ovelha durante a prenhez ou a lactação são substancialmente maiores que em outros estádios fisiológicos. Portanto, o baixo aporte energético na prenhez pode ter resultado no nascimento de cordeiros menos pesados e menos viáveis (SEEGERS e DENIS 1982; THERIEZ et al., 1987; e FIGUEIRÓ, 1989).

MACEDO (1983) encontrou em um rebanho Morada Nova, no Estado do Ceará, taxa de mortalidade de cordeiros até o desmame de $22,22 \%$, incidindo principalmente sobre os animais menos pesados ao nascer ou oriundos de partos múltiplos. Comparado aos resultados do presente estudo, o desempenho dos cordeiros $\mathrm{F}_{1}$ assemelhou-se ao de cordeiros de raças já adaptadas a ambientes tropicais semi-áridos.

As menores taxas de sobrevivência de crias, quando os partos ocorreram na época seca de 1988 ou na chuvosa de 1989 , podem ter razões distintas. A carência de suficiente substrato alimentar na época seca pode ter prejudicado o desempenho das matrizes recém paridas e de suas crias, o que corrobora as conclusões de FIGUEIRÓ (1989). Por outro lado, a maioria dos nascimentos em 1989 coincidiu ou foi imediatamente posterior a precipitações pluviais, da ordem de $751,0 \mathrm{~mm}$, ocorridas entre março e maio (MACHADO e SIMPLÍCIO, 1998), com queda da temperatura média e aumento do gradiente térmico entre o dia e a noite. Estas intempéries favoreceram a mortalidade de crias por pneumonias e outras afecções oriundas da menor competência termo-reguladora de mamíferos jovens (ENCARNAÇÃO, 1986; FIGUEIRÓ, 1989) e do eventual favorecimento à proliferação de patógenos em habitats com elevadas temperatura e umidade relativa do ar.

A época de monta não influenciou significativa-

Tabela 4 - Efeito da época de parição sobre a eficiência produtiva de ovelhas deslanadas de raça nativa Table 4 - Effect of season of lambing on the productive performance of native hairsheep ewes

\begin{tabular}{|c|c|c|c|c|c|c|c|c|}
\hline \multirow[t]{3}{*}{$\begin{array}{l}\text { Epoca } \\
\text { Season }\end{array}$} & \multicolumn{2}{|c|}{$\begin{array}{c}\text { Taxa }(\%) \\
\text { Rate }\end{array}$} & \multirow[t]{3}{*}{$\mathrm{N}$} & \multicolumn{2}{|c|}{$\begin{array}{l}\text { Peso das crias } \\
\text { Lamb weight }\end{array}$} & \multirow{3}{*}{$\begin{array}{c}\text { Ganho de peso } \\
\text { no aleitamento }(\mathrm{g} / \mathrm{d}) \\
\text { Daily gain } \\
\text { at suckling }\end{array}$} & \multirow{2}{*}{\multicolumn{2}{|c|}{$\begin{array}{c}\text { Peso total } \\
\text { de cordeiro }(\mathrm{kg}) \\
\text { Total weight of lambs }\end{array}$}} \\
\hline & Desmama & Sobrevivência & & Ao nascer & Ao desmame & & & \\
\hline & Weaning & Survival & & At birth & Atweaning & & $\begin{array}{c}\text { Nascido } \\
\text { Born }\end{array}$ & $\begin{array}{c}\text { Desmamado } \\
\text { Weaned }\end{array}$ \\
\hline set- nov/88 & 93,7 & $81,1^{b}$ & 96 & $3,14^{b}$ & $8,44^{\mathrm{c}}$ & $63,4^{\mathrm{c}}$ & 5,71 & $8,84^{\mathrm{c}}$ \\
\hline mai-jul/89 & 92,9 & $82,2^{b}$ & 100 & $3,91^{\mathrm{a}}$ & $10,79^{b}$ & $83,0^{\mathrm{b}}$ & 5,69 & $10,62^{b}$ \\
\hline mar-abr/90 & 94,2 & $94,9^{\mathrm{a}}$ & 106 & $3,94^{\mathrm{a}}$ & $17,70^{\mathrm{a}}$ & $165,8^{\mathrm{a}}$ & 5,90 & $19,86^{\mathrm{a}}$ \\
\hline
\end{tabular}

Médias, na coluna, seguidas de letras diferentes são diferentes $(P<0,05)$ pelo teste LSD.

Means, within a column, followed by different letters are different $(P<.05)$ by $L S D$ test. 
mente o peso vivo total das crias ao nascer, mesmo quando os partos ocorreram no período mais quente do ano (setembro a novembro), contrariando assim os achados de SEEGERS e DENIS (1982), CUELLAR e MUÑOZ (1986) e CONSTANTINOU e MAVROGENIS (1987). As diferenças “entre épocas" na prolificidade explicam este fenômeno, pois fêmeas paridas na época seca haviam sido acasaladas na época chuvosa, permitindo a ocorrência de maior número de ovulações por fêmea exposta (SILVA et al., 1987) e compensando o menor desenvolvimento fetal das acasaladas entre abril e julho.

Os efeitos do tipo de nascimento, do sexo da cria e da época de nascimento sobre o desenvolvimento ponderal dos cordeiros lactentes e a sua viabilidade constam da Tabela 5. As crias de nascimento simples foram mais pesadas que as de nascimento duplo ao longo de todo o período de aleitamento - resultado similar ao obtido por FERNANDES (1985), FALAGAN e GARCIA DE SILES (1986), CONSTANTINOU e MAVROGENIS (1987) e SILVA (1990).

MACEDO (1983) verificou taxas de mortalidade de 15,66 e 26,82\% para cordeiros de nascimentos simples e duplos, respectivamente. FERNANDES (1985) também relatou efeito do tipo de nascimento sobre a mortalidade. Os achados deste trabalho, embora não-significativos, apresentaram esta mesma tendência, com taxas de 15,93 e 23,08\%, respec- tivamente, para crias de nascimentos simples e duplos. As crias de sexo feminino, oriundas de nascimentos simples tenderam a obter as maiores taxas de sobrevivência, concordando com os achados de FERNANDES (1985). Os resultados do presente estudo estão em concordância com o relato de SILVA (1990), que, ao estudar mestiços de Santa Inês e SRD, não verificou efeito do sexo da cria sobre os pesos até o desmame e sobre a taxa de mortalidade, discordando, entretanto, dos relatos de FERNANDES (1985) com Morada Nova e FALAGAN e GARCIA DE SILES (1986) com cruzas da raça Aragonesa.

A viabilidade e o desempenho ponderal dos cordeiros foram influenciados $(\mathrm{P}<0,01)$ pela época do nascimento. A maior disponibilidade quanti-qualitativa de forragens para as matrizes paridas na época chuvosa, com conseqüente aumento no aporte energético, possivelmente favoreceu a produção leiteira das ovelhas, permitindo ganhos de peso e viabilidade de crias elevados (FIGUEIRÓ, 1989). Os relatos de GARCIA et al. (1984) e CUELLAR e MUÑOZ (1986) confirmam esta hipótese, em razão dos resultados desses terem sido similares aos do presente trabalho, nas respectivas épocas de abundância ou carência de forragens. Em adição, o crescimento do cordeiro nas primeiras seis semanas de vida é, principalmente, determinado pela quantidade de leite fornecida da mãe. Desse modo, a melhor oferta

Tabela 5 - Peso vivo médio $(\mathrm{kg})$ e taxa de mortalidade de cordeiros $\mathrm{F}_{1}$ mestiços, de acordo com o tipo de nascimento, o sexo e a época de nascimento

Table 5 - Mean live weight $(\mathrm{kg})$ and mortality rate of crossbred lambs according to type of birth, sex and season of lambing

\begin{tabular}{|c|c|c|c|c|c|c|}
\hline & \multicolumn{5}{|c|}{$\begin{array}{c}\text { Peso vivo (kg) } \\
\text { Live weight }\end{array}$} & \multirow[t]{3}{*}{$\begin{array}{c}\text { Mortalidade }(\%) \\
\text { Mortality }\end{array}$} \\
\hline & Nascer & 28 dias & 56 dias & 84 dias & 112 dias & \\
\hline & Birth & 28 days & 56 days & 84 days & 112 days & \\
\hline \multicolumn{7}{|l|}{ Nascimento } \\
\hline \multicolumn{7}{|l|}{ Type of birth } \\
\hline Simples & $3,92 * *$ & $8,07 * *$ & $10,51 * *$ & $13,39 * *$ & $17,44 * *$ & 15,93 \\
\hline Single & & & & & & \\
\hline Duplo & 2,98 & 5,37 & 7,90 & 10,24 & 14,53 & 23,08 \\
\hline Twin & & & & & & \\
\hline \multicolumn{7}{|l|}{ Sexo } \\
\hline \multicolumn{7}{|l|}{ Sex } \\
\hline Macho & 3,74 & 7,79 & 10,17 & 13,05 & 16,72 & 20,71 \\
\hline Ram lamb & & & & & & \\
\hline Fêmea & 3,63 & 7,14 & 9,66 & 12,27 & 16,84 & 15,10 \\
\hline Ewe lamb & & & & & & \\
\hline \multicolumn{7}{|l|}{ Época } \\
\hline \multicolumn{7}{|l|}{ Season } \\
\hline set - nov $/ 88$ & $3,14 * *$ & $5,87 * *$ & $7,50 * *$ & $8,43 * *$ & - & $18,92 * *$ \\
\hline mai-jul/89 & 3,91 & 7,87 & 9,21 & 10,79 & $12,96 * *$ & 27,82 \\
\hline $\mathrm{mar}-\mathrm{abr} / 90$ & 3,94 & 8,49 & 12,58 & 17,70 & 19,85 & 5,13 \\
\hline
\end{tabular}


712 Rev. bras. zootec.

nutricional durante o aleitamento determina redução na mortalidade das crias e ritmos de crescimento elevados (FIGUEIRÓ, 1989), pois a curva de crescimento do cordeiro na fase de aleitamento acompanha a curva de lactação da matriz (FIGUEIRÓ, 1989).

\section{Conclusões}

Os acasalamentos efetuados entre os meados e o final da época chuvosa proporcionaram taxas produtivas mais baixas, em função de a prenhez ser desenvolvida e posta a termo durante a época de carência alimentar na região.

Os efeitos da heterose não foram quantificados por carência de um grupo controle fidedigno (machos sem raça definida acasalados com fêmeas sem raça definida). No entanto, a velocidade de crescimento e a alta viabilidade dos cordeiros $\mathrm{F}_{1}$ Santa Inês até a desmama não diferiram das obtidas por crias de raças especializadas para corte, permitindo recomendar a inclusão deste grupo genotípico como raça parental em programas de cruzamento industrial desenvolvidos na região Nordeste do Brasil.

\section{Referências Bibliográficas}

ABOUL-NAGA, A.M., ABOUL-ELA, M.B. 1987. Performance of subtropical Egyptian breeds of sheep, European breeds and their crosses. World Rev. Anim. Prod., 23(3):9-15.

BUIRAS, E., ESTEVA, J., PICARDI, L. 1986. Peso al nacimiento y eficiencia pre-destete en corderos de la raza Ideal y su cruza con Hampshire Down y Texel. Rev. Argentina Prod. Anim., 6(9-10):581-586.

CARDELLINO, R.A. Sistemas de produção de carne ovina utilizando cruzamentos. In: SIMPÓSIO PAULISTA DE OVINOCULTURA, 1, 1988. Botucatu. Anais. Campinas: Fundação Cargill, 1989. p.97-104.

CASOLI, C., DURANTI, E., BIANCHI, R.F. et al. 1984. Provi di incrocio industriale su pecore di razza Appenninica. Zootec. Nut. Anim., 10(3):217-222.

CONSTANTINOU, A., MAVROGENIS, A.P. Environmental, breed and heterosis effects on lamb mortality and weight in Chios, Awassi and reciprocal crossbreed sheep. In: INTERNATIONAL CONFERENCE ON ANIMAL PRODUCTION IN ARID ZONES, 2, 1987. Proceedings... Syria: Arab Center for the studies of Arid Zones and Drylands, Syria, 1987. p.705-717.

CUELLAR, O.J.A., MUÑOZ, H.J.C. Influencia de la época de parto en el peso al nacimiento y mortalidad de corderos criollos. In: REUNION DE INVESTIGACIÓN PECUARIA EN MÉXICO, 1986. Mexico City. Memorias... Mexico City: Talleres de Impresora Bravo, 1986. p.172.

ENCARNAÇÃO, R.O. 1986. Estresse e produção animal. Campo Grande, EMBRAPA-CNPGC, 32p. (EMBRAPACNPGC. Documentos, 34).

FALAGAN, A., GARCIA DE SILES, J.L. 1986. Influencia de la raza paterna en la producción de corderos procedentes de cruzamientos industriales con "rasa Aragonesa". I. Caracteristicas de crescimiento. Investigación Agraria,
Producción y Sanidad Animales, 1(1-2):11-23.

FERNANDES, A.A.O. Genetic and environmental factors affecting growth and reproduction characters of Morada Nova sheep in Northeast Brazil. Texas: Texas A\&M University, 1985, 86p. Thesis (Master).

FERNANDES, A.A.O., FIGUEIREDO, E.A.P., SHELTON, J.M. et al. Performance of Morada Nova sheep. In: REUNIÃO TÉCNICO-CIENTï'FICA DO PROGRAMA DE APOIO À PESQUISA COLABORATIVA DE PEQUENOS RUMINANTES, 1. Sobral, 1986. Proceedings... Sobral: EMBRAPA-CNPC/SR-CRSP, 1986. p.327-46. (EMBRAPA-CNPC/ Documentos,6).

FERNANDES, A.P., DESHMUKH, A.P. 1988. Effect of various factors of reproduction on the birth weight of lambs born to Deccani and its crosses. Livestock Adviser, 13(2):20-4.

FIGUEIRÓ, P.R.P. Manejo alimentar de ovinos. In: SIMPÓSIO PAULISTA DE OVINOCULTURA,1, 1988. Botucatu. Anais... Campinas: Fundação Cargill, 1989. p. 22-33.

GARCIA, D.G., OLIVARES, E.A., GARCIA, F.X. et al. Un sistema de produccion semi-intensivo para ovinos Suffolk en la región Central. In: INVESTIGACIÓN DEL DEPARTAMIENTO DE PRODUCCIO'N ANIMAL, 1981/1982. Santiago. Proceedings... Santiago: Universidad de Chile, 1984. p.96-102.

GUERREIRO, J.L.V. Programa de melhoramento para ovinos de raças mistas (lã + carne). In: SIMPÓSIO PAULISTA DE OVINOCULTURA, 1, 1988. Botucatu. Anais... Campinas: Fundação Cargill, 1989. p. 80-96.

GUNEY, O. 1990. Commercial crossbreeding between Ile-deFrance, Rambouile, Chios and local fat-tail Awassi for market lamb production. Small Ruminant Research, 3(5):449-56.

MACÊDO, F.A.R. Alguns fatores que afetam o comportamento reprodutivo e produtivo de ovinos da raça Morada Nova, variedade vermelha. Santa Maria: UFSM, 1983. 91p. Dissertação (Mestrado em Zootecnia) - Universidade Federal de Santa Maria, 1983.

MACHADO, R., SIMPLÍCIO, A.A. 1998. Efeito da raça do padreador e da época de monta sobre a eficiência reprodutiva de ovelhas deslanadas acasaladas com reprodutores de raças especializadas para corte. R. Bras. Zootec., 27(1):54-59.

McEWAN, J.C., HANRAHAN, J.P. Texel and Suffolk growth patterns. In: MOOREPARK AND WESTERN RESEARCH CENTRES, 1986. Dublin. Report from Grange/Dunsinea. Dublin: Animal Production Series. 1986. 23p.

SAS - STATISTICAL ANALYSIS SYSTEM. SAS®. USER'S GUIDE: Statistical, ver. 6.0.3.ed. Cary, N.C.: SAS Institute Inc., Cary, 1990.

SEEGERS, H., DENIS, B. 1982. Facteurs zootechniques et pèrtes périnatales en élevage ovin. Recuell Médique Véterinarie, 158(2):431-40.

SILVA, A.E.D.F., FOOTE, W.C., RIERA, G.S. et al. 1987. Efeito do manejo nutricional sobre a taxa de ovulação e de folículos no decorrer do ano, em ovinos deslanados no Nordeste do Brasil. Pesq. Agropec. Bras., 22(6):635-45.

SILVA, F.L.R. da . Efeito de fatores genéticos e de ambiente sobre o desempenho de ovinos mestiços Santa Inês, no Estado do Ceará. Viçosa: UFV, 1990. 93p. Dissertação (Mestrado em Zootecnia) - Universidade Federal de Viçosa, 1990.

STEEL, R.G.D., TORRIE, J.H. 1980. Principles and Procedures in Statistics: A Biometrical Approach. 2. ed. New York: McGraw-Hill Book Co., 174p.

THERIEZ, M., BOCQUIER, F., BRELURUT, A. 1987. Recommendations alimentaires pour les brebis à lèntretien et en gestation. Bulletin Technique C.R.Z.V. Theix-INRA, 70(4):185-97.

Recebido em: 07/01/97

Aceito em: 03/02/99 